\title{
2006-2016: UNA DÉCADA DEL PROGRAMA DE ESTUDIOS BÁSICOS Y DE EXPLOTACIÓN DE RECURSOS NATURALES EN EL PERÚ
}

\author{
Juvenal Cabezas Oruna \\ Universidad Ricardo Palma \\ juvecabezas@yahoo.com
}

\section{RESUMEN}

El Programa de Estudios Básicos de la Universidad Ricardo Palma está cumpliendo a mediados de este año los primeros diez años de vida (2006-2016). El curso Recursos Naturales y Ecología, hoy Recursos Naturales y Medio Ambiente, ha acompañado al Programa de Estudios Básicos en toda esta década, desde su inicio, dictándose a todos los alumnos de la universidad. Una de las unidades temáticas principales del curso es el estudio de los recursos naturales que se dicta en las primeras semanas y que constituye un tema muy sensible en el país debido al impacto que tiene la explotación de los recursos naturales tanto en la economía nacional, como en las diversas comunidades peruanas y en el medio ambiente. Por este motivo el autor incluye algunas apreciaciones personales relacionadas con las decisiones que se han tomado alrededor de nuestros recursos durante los dos gobiernos democráticos desarrollados en dicha década correspondientes a los periodos 2006-2011 y 20112016. En el presente artículo se revisa la explotación durante los años 2006-2016 de los siguientes recursos naturales: minerales, petróleo, gas natural, energía hidráulica, energía eólica, energía solar y roca fosfórica.

\section{PALABRAS CLAVE}

Recursos naturales/ Ecología/ Medio ambiente/ Explotación/ Impacto/ Comunidades/ Gobierno

\section{ABSTRACT}

Towards the middle of this year, the Basic Studies Program of Ricardo Palma University celebrated its first ten years of existence (2006-2016). The course of Natural Resources and Ecology, today called Natural Resources and Environment, has accompanied the Basic Studies Program along this decade, and has being imparted to all of the students of the university. One of the main thematic units of 
the course is the study of the natural resources, which is studied in the first weeks and that constitutes a very sensitive topic in the country due to the impact that natural resources extract has on the national economy, as well as among the different Peruvian communities and the environment. It is because of this, that the author includes a personal perspective related to the decisions that had been taken around the natural resources during the two democratic governments correspondent to the 2006-2011 and 2011-2016 periods. In this article, the exploitation during the decade 2006-2016 is reviewed within the following natural resources: minerals, petroleum, natural gas, hydraulic energy, eolic energy, solar energy and phosphoric rock.

\section{KEY WORDS}

Natural resources/ Ecology/ Environment/ Exploitation/ Impact/ Communities/ Government

\section{INTRODUCCIÓN}

La Universidad Ricardo Palma celebra este año con mucha alegría la primera década de funcionamiento del Programa de Estudios Básicos. Uno de los cursos que ha acompañado al Programa de Estudios Básicos desde sus inicios en el segundo semestre del año 2006 ha sido el curso Recursos Naturales y Ecología, que el año pasado cambió su nombre a Recursos Naturales y Medio Ambiente. Este curso ha sido dictado ininterrumpidamente a todos los alumnos de la universidad durante estos 10 años teniendo como uno de los temas centrales el estudio de los recursos naturales. Este tema es muy sensible en el Perú debido al impacto que tiene la explotación de los recursos naturales no solamente en la economía del país, sino también sobre otras dimensiones del desarrollo sostenible como son el componente social y el componente ambiental.

El objetivo de este artículo es hacer una revisión de la forma en que se ha llevado a cabo la explotación de los recursos naturales en nuestro país en la década que corre paralela a la existencia del Programa de Estudios Básicos. Es importante remarcar también que en esta década hemos tenido dos gobiernos constitucionales en el Perú: la de los presidentes Alan García Pérez del 2006 al 2011 y de Ollanta Humala Tasso del 2011 al 2016. Por la connotación del tema a desarrollar (los recursos naturales de nuestro país) es inevitable hacer referencias y comentarios puntuales a algunas de las decisiones de estos gobiernos con relación a la explotación de los recursos naturales. El presente artículo revisa la explotación, durante los años 2006-2016, de los siguientes recursos naturales: minerales, petróleo, gas natural, energía hidráulica, energía eólica, energía solar y roca fosfórica. 


\section{MINERALES}

La fluctuación de los precios internacionales ha marcado el auge y la caída de la producción peruana de metales en los años 2006-2016. Inicialmente y respondiendo a factores externos, entre los que está principalmente el crecimiento de la economía china, la explotación de minerales en el Perú se incrementó notablemente en la década pasada alcanzando niveles de producción y exportación no vistos anteriormente. Paralelamente los ingresos de las arcas fiscales también se incrementaron fuertemente. Pero no se aprovechó esta bonanza para poner los cimientos de un país con mayor desarrollo futuro, manteniéndose por ejemplo la pobre industrialización del país, que en este caso no le da mayor valor agregado a su producción minera, exportando en algunos casos solamente los concentrados. Así, en el caso del hierro no se aprovecha hasta ahora el gas de Camisea para convertir los pellets de mineral de hierro en hierro esponja que se usa para producir acero. Asimismo, tanto el gobierno central, como los gobiernos regionales y municipales no han dado un uso adecuado al canon y otros ingresos derivados de las exportaciones mineras durante los años 2006 al 2011. Fueron años de bonanza desperdiciada debido a que, a comienzos de la presente década, China disminuyó sus niveles de desarrollo y los países europeos entraron en graves problemas económicos, de los que aún no se recuperan, y los precios internacionales de los metales empezaron a disminuir mucho llegando a niveles preocupantes en el año 2015. Se estima que esta situación mejorará, aunque muy ligeramente hacia los años 2018-19. En la Tabla $\mathrm{N}^{\circ} 1$ se nota la influencia de la actividad minera en la economía nacional así como la caída que ha tenido en los dos últimos años. La Tabla ํ2 muestra el impacto de los precios sobre las exportaciones, notándose que la variación a la baja entre los años 2012 y 2013 fue notable para el cobre, oro y plomo.

TABLA $\mathrm{N}^{\circ} 1$

EXPORTACIONES MINERAS VS. EXPORTACIONES TOTALES DEL PERÚ

\begin{tabular}{|c|c|c|c|c|c|}
\hline Indicador & $\mathbf{2 0 0 9}$ & $\mathbf{2 0 1 0}$ & $\mathbf{2 0 1 1}$ & $\mathbf{2 0 1 2}$ & $\mathbf{2 0 1 3}$ \\
\hline $\begin{array}{c}\text { Exportaciones mineras } \\
\text { MM US\$ }\end{array}$ & 16361 & 21743 & 27361 & 25921 & 23030 \\
\hline $\begin{array}{c}\text { Exportaciones totales } \\
\text { MM US\$ }\end{array}$ & 26962 & 35565 & 42268 & 45639 & 41826 \\
\hline$\%$ & 60.7 & 61.1 & 64.7 & 56.8 & 55.1 \\
\hline
\end{tabular}

Fuente: MINISTERIO DE ENERGÍA Y MINAS. Boletín Estadistico de Minería, Reporte Anual. Lima: MINEM, 2013. p. 2 - 11. 
TABLA N ${ }^{\circ} 2$

EXPORTACIONES MINERAS E IMPACTO DE LOS PRECIOS

\begin{tabular}{|c|c|c|c|c|c|c|c|c|}
\hline Metal & Item & Unidad & $\mathbf{2 0 0 6}$ & $\mathbf{2 0 0 8}$ & $\mathbf{2 0 1 0}$ & $\mathbf{2 0 1 2}$ & $\mathbf{2 0 1 3}$ & Variación \\
& & & & & & & & $\mathbf{2 0 1 3 / 1 2}$ \\
\hline COBRE & Valor & MMUS\$ & 5996 & 7277 & 8870 & 10728 & 9809 & $-8.6 \%$ \\
\hline & Cantidad & M TM & 981 & 1243 & 1254 & 1405 & 1401 & $-0.2 \%$ \\
\hline & Precio & Ct \$ / lb & 275 & 272 & 321 & 346 & 318 & $-8.3 \%$ \\
\hline & & & & & & & & \\
\hline ORO & Valor & MMUS\$ & 4032 & 5586 & 7756 & 9594 & 7846 & $-18.2 \%$ \\
\hline & Cantidad & M Oz Tr & 6674 & 6418 & 6346 & 5740 & 5555 & $-3.2 \%$ \\
\hline & Precio & \$ OzTr & 605 & 873 & 1225 & 1670 & 1412 & $-15.5 \%$ \\
\hline & & & & & & & & \\
\hline PLOMO & Valor & MMUS\$ & 713 & 1136 & 1579 & 2575 & 1749 & $-32.1 \%$ \\
\hline & Cantidad & M TM & 378 & 525 & 770 & 1170 & 847 & $-27.6 \%$ \\
\hline & Precio & Ct \$ / lb & 83 & 100 & 92 & 100 & 94 & $-6.5 \%$ \\
\hline
\end{tabular}

Fuente: MINISTERIO DE ENERGÍA Y MINAS. Boletín Estadístico de Minería, Reporte Anual. Lima: MINEM, 2013. p. 2 - 11.

En la década a la que hacemos referencia 2006-2016, la explotación minera se ha mantenido también como la actividad que genera la mayor cantidad de conflictos sociales y ambientales. Los ejemplos más notables se han sucedido a lo largo de estos diez años han sido: primero la operación de la mina Yanacocha en Cajamarca y su deseo de ampliar su presencia mediante el proyecto 'Conga', que originó violentas protestas incluso en la capital departamental. Segundo, la operación contaminante del Complejo Metalúrgico de La Oroya en Junín y la actuación de la empresa Doe Run, que ha originado constantes bloqueos de la carretera central. Tercero, el Proyecto minero Tía María en la costa de Arequipa, que también ha tenido violentas protestas y fallecidos, en donde el uso del agua no fue bien planteado en el Estudio de Impacto Ambiental. Cuarto, la operación de la mina Las Bambas en Apurímac junto con la mina Tintaya en Espinar-Cusco, ambas de la empresa china MMG, etc. En todos estos conflictos hay un denominador común: la preocupación de las poblaciones locales respecto a la situación del recurso agua y la contaminación de su ambiente.

En las convenciones mineras internacionales se señala constantemente que el Perú es el tercer productor mundial de cobre, plata, zinc y estaño y el sexto productor de oro. Esta presencia mundial está en peligro de perderse por los 
conflictos mineros que se han dado debido al deficiente trabajo de las mineras, que ha dejado cientos de minas abandonas llamadas 'pasivos ambientales' que no han sido remediadas en la década 2006-2016, empresas que han contaminado y tratado mal a las comunidades, y que ha originado que ahora éstas se muestren contrarias a dar la licencia social a los nuevos proyectos. Por este motivo los proyectos auríferos, entre otros, no se están desarrollando a la velocidad deseada y la producción de las minas que están en operación va decayendo año a año de manera natural. Por ejemplo, hasta el año 2012 Cajamarca era el departamento con la mayor producción de oro, pero ya no lo es más; actualmente el departamento que produce más oro en el Perú es La Libertad. Esto se debe a que Yanacocha y otras minas de Cajamarca han ido decayendo en su producción y no se ha cristalizado el proyecto Conga, mientras que en La Libertad se ha incrementado un poco la producción de oro especialmente en las minas Laguna Grande (Barrick), La Arena (Río Alto) y Quiruvilca (Pan American).

\section{PETRÓLEO}

Es conocido que nuestro país produce actualmente poco petróleo en Talara (Piura), Contralmirante Villar (Tumbes), Selva Norte (Loreto) y Selva Central (Ucayali) y que la producción actual no cubre nuestras necesidades, obligando a importar alrededor de cien mil barriles diarios de crudo y derivados. A comienzos de la década del 90 éramos autosuficientes con una producción cercana a ciento treinta mil barriles diarios, pero con la privatización impuesta por el gobierno de turno la producción cayó paulatinamente hasta los niveles actuales de cincuenta mil barriles diarios. La famosa privatización de entonces solo sirvió a algunos intereses, no trajo la tecnología y capitales que se mencionaron en esos años y no se descubrieron nuevas estructuras petroleras significativas hasta la fecha. La Tabla $\mathrm{N}^{\circ}$ 3 muestra la forma como la producción petrolera ha caído durante los años 20062016, años de existencia del Programa de Estudios Básicos en nuestra Universidad. 
TABLA $\mathrm{N}^{\circ} 3$

PRODUCCIÓN DE PETRÓLEO CRUDO EN EL PERÚ - AÑOS 2006/2016

\begin{tabular}{|c|c|}
\hline AÑO & PRODUCCIÓN BARR/DÍA \\
\hline 1994 & 127440 \\
\hline 2006 & 77500 \\
\hline 2007 & 77100 \\
\hline 2008 & 76577 \\
\hline 2009 & 71032 \\
\hline 2010 & 72690 \\
\hline 2011 & 69550 \\
\hline 2012 & 66650 \\
\hline 2013 & 62900 \\
\hline 2014 & 69300 \\
\hline 2015 & 58010 \\
\hline 2016 & 48820 (a agosto) \\
\hline
\end{tabular}

Fuente: PERUPETRO. Estadistica Petrolera. Lima, 2016.

De manera similar a la industria minera, en los últimos diez años la industria petrolera ha sido origen de numerosos conflictos sociales con las poblaciones locales, que han visto cómo sus comunidades han sido invadidas, sus fuentes de agua contaminadas, sus alimentos afectados, etc. La mayor cantidad de conflictos se originan por la contaminación de suelos y aguas de las comunidades debido a fugas y derrames de petróleo y de agua de formación ${ }^{1}$, que van a dar al suelo y a los ríos de la amazonia. El mayor conflicto de la última década se dio el 5 de julio de 2009 en Bagua, departamento de Amazonas, muy cerca a la Estación 7 del Oleoducto Norperuano, en donde murieron 10 nativos y 23 policías durante el desalojo de una numerosa población que había tomado la carretera Fernando Belaunde durante casi 2 meses en protesta por las actividades petroleras en sus comunidades.

Ahora se está incubando otro conflicto al oriente de Bagua, en las cuencas de los ríos Pastaza, Corrientes, Tigre y Marañón en la selva norte del departamento de Loreto, originado principalmente por el incumplimiento de los acuerdos de marzo del año 2015 entre el gobierno y cuatro federaciones indígenas luego de cuatro

1 El agua de formación es el agua que se ha formado con el petróleo en el yacimiento y que es sacada a la superficie junto con el petróleo durante la fase de explotación del yacimiento. 
décadas de explotación petrolera. Estos acuerdos pendientes de cumplir abarcan la ejecución de la remediación ambiental de los territorios, la aplicación de la consulta previa, la titulación de las tierras comunales, el respeto de los territorios comunales vírgenes, y mejorar el control de la operación de los yacimientos, oleoductos y de las barcazas en los ríos para evitar derrames.

En la década del 2006 al 2016 las mayores inversiones petroleras se han dado en los últimos años con la modernización de la refinería de Talara que tiene $40 \%$ de avance y la construcción de nuevas unidades de conversión y de desulfurización de diésel y naftas en refinería La Pampilla que han entrado en operación este año. En las otras cinco refinerías no han habido inversiones significativas, de tal manera que no ha mejorado la calidad de los combustibles obtenidos en estas refinerías, principalmente la concentración de azufre en el diésel y en la nafta. Por este motivo en los últimos años la calidad de los combustibles se ha basado en naftas y diesel importados y en el diésel adquirido a la planta de fraccionamiento de los líquidos del gas natural de Paracas, así como a la inclusión de bioetanol y biodiesel en los gasoholes y diésel B5 respectivamente. Se espera que el proyecto de modernización de la refinería de Talara no sufra retrasos para que dentro de tres años tengamos combustibles mucho más limpios para nuestra industria y para el parque automotor.

\section{GAS NATURAL}

Las zonas productoras de gas natural siguen siendo Camisea (Cusco), Talara (Piura), Contralmirante Villar (Tumbes) y Aguaytía (Ucayali). Luego de entrar en producción Camisea el año 2005, en los últimos diez años solo se ha encontrado un poco más de gas en áreas cercanas a Camisea, pero lo más significativo es que los volúmenes de extracción han ido incrementándose de manera notable en la década 2006-2016 debido a la ampliación de todo el proyecto para atender los contratos de exportación del gas, tal como se observa en la Tabla $\mathrm{N}^{\circ} 4$ que muestra la producción de gas seco y de líquidos del gas natural en el Perú. 
TABLA N ${ }^{\circ} 4$

PRODUCCIÓN DE GAS Y LGN EN EL PERÚ - AÑOS 2006/2016

\begin{tabular}{|c|c|c|}
\hline AÑO & $\begin{array}{c}\text { PRODUCCIÓN DE GAS } \\
\text { MILLONES PIES } / \text { /DÍA }\end{array}$ & $\begin{array}{c}\text { PRODUCCIÓN DE LGN } \\
\text { BARRILES/DÍA }\end{array}$ \\
\hline 2006 & 171.70 & 38.00 \\
\hline 2007 & 258.90 & 36.80 \\
\hline 2008 & 327.75 & 43.45 \\
\hline 2009 & 336.11 & 74.25 \\
\hline 2010 & 700.30 & 84.47 \\
\hline 2011 & 1099.09 & 83.16 \\
\hline 2012 & 1144.25 & 86.33 \\
\hline 2013 & 1179.61 & 104.62 \\
\hline 2014 & 1250.43 & 103.43 \\
\hline 2015 & 1208.89 & 91.40 \\
\hline 2016 & 1495.09 (a agosto) & 106.61 (a agosto) \\
\hline
\end{tabular}

Fuente: PERUPETRO. Estadistica Petrolera. Lima, 2016.

Como sabemos, el gas de Camisea es húmedo, es decir tiene dos fases: contiene gases (metano y etano) y también hidrocarburos líquidos que dan lugar a los condensados o líquidos del gas natural (LGN). La separación del gas de Camisea en estas dos fases se hace en la localidad de Malvinas, provincia de La Convención, ubicada en la selva baja del Cusco, y desde allí salen a la costa dos ductos: un ducto llamado gasoducto que conduce el 'gas' (que es una mezcla de $92 \%$ de metano y $8 \%$ de etano) que va a la costa para ser exportado y para ser usado en los mercados de Lima, Callao e Ica. El otro ducto conduce los LGN y termina en Pisco, muy cerca de Paracas, en donde se destilan los líquidos en una planta de fraccionamiento para obtener propano, butano, nafta petroquímica y diésel.

Las características del contrato firmado entre el gobierno peruano y las empresas internacionales operadoras de Camisea no permiten que el gobierno peruano pueda disponer hasta la fecha del gas natural porque la Ley 26221 de 1993 establece que los propietarios de los hidrocarburos son los que tienen el contrato de licencia, previo pago de una regalía. (Campodónico, H. 2016, p. 5). Y con esta base en la década 2006-2016 se ha privilegiado la exportación de esta gran riqueza nacional. A lo largo de estos años la infraestructura original del proyecto Camisea (450 millones de pies cúbicos diarios para gas y 85 mil barriles diarios 
para líquidos) se ha ampliado rápidamente con dicho fin hasta 1,160 millones de pies cúbicos diarios de gas en Malvinas y 120 mil barriles diarios para líquidos en Paracas. (Tamayo, J. 2014. p. 62 y 64). El incremento de los volúmenes producidos mostrados en la Tabla $\mathrm{N}^{\circ} 4$ lo dicen claramente: son para la exportación.

Debido a la prioridad que se le ha dado a la exportación, en estos años no se han ejecutado proyectos de industrialización aprovechando el gas natural, por ejemplo en la producción de urea, un gran fertilizante que es importado en su totalidad, o en la producción de hierro esponja, etc. No solamente se privilegia la exportación del gas; también de un derivado importante de los líquidos destilados en Paracas, como es la nafta petroquímica, que no se aprovecha en el país. Por otro lado, el propano y el butano que se obtienen en Paracas se mezclan entre sí para obtener gas licuado de petróleo (GLP) y así se venden en el mercado interno para usarse como combustible residencial y automotor. Pero otra forma de aprovechar mucho mejor el propano y el butano, y darles mayor valor agregado, es como materia prima en la industria petroquímica para la producción de olefinas como el etileno, que es a su vez la materia prima principal para producir polietileno, PVC y otros. O también para obtener propileno y otras olefinas primarias que dan lugar a la producción de docenas de compuestos petroquímicos intermedios y finales, que son productos que no producimos en el país.

\section{ENERGÍAS RENOVABLES}

El Perú tiene un enorme potencial para desarrollar estas energías a una velocidad mayor a la que se está dando en los últimos años. En el caso de la Hidroelectricidad producida por caída de agua, tenemos unas 40 hidroeléctricas que solamente aprovechan el $5 \%$ del potencial eléctrico por caída de agua en el país. De tal manera que incluso duplicando el número de centrales solo llegaríamos a aprovechar el $10 \%$ del potencial total. Por este motivo se podrían construir en el corto y mediano plazo más centrales hidroeléctricas de gran potencia (más de 10 MW) para alimentar al sistema interconectado nacional, así como minicentrales hidráulicas (entre 1 y $10 \mathrm{MW}$ ) y microcentrales hidroeléctricas (menos de $1 \mathrm{MW}$ ) para proporcionar electricidad directamente a pequeñas poblaciones aprovechando nuestros ríos y el gran desnivel de nuestro territorio por la presencia de la Cordillera de los Andes. En la última década destaca la construcción de la Central Hidroeléctrica Chaglla en Huánuco, a unos 50 kilómetros de Tingo María, que represa las aguas del río Huallaga para generar $450 \mathrm{MW}$ de electricidad. El mayor aprovechamiento del potencial hidroeléctrico en el Perú se debe hacer primero elaborando excelentes estudios de impacto ambiental para no afectar a las poblaciones locales y su entorno, de tal manera que el impacto sea mínimo. De esta manera podríamos generar más 
electricidad para entregarla a las poblaciones del país e incluso venderla a nuestros vecinos, especialmente a Brasil y Chile que han manifestado continuamente su deseo de ser abastecidos de energía.

Paralelamente a los diez años de vida del Programa de Estudios Básicos se ha empezado a producir electricidad en el Perú, en gran escala, aprovechando la fuerza del aire y la energía solar. En el caso de la energía eólica han entrado en operación cuatro parques eólicos desde el año 2014, ubicados en los departamentos de Ica (2) y La Libertad y Piura que aportan $240 \mathrm{MW}$ al sistema eléctrico nacional. El potencial eólico aprovechable del Perú es de 22,450 MW, (MINEM, 2008. p. 16), de tal manera que el potencial que tenemos para seguir desarrollando esta energía en los siguientes años es enorme.

Los parques eólicos que ya están operando son: 'Cupisnique' en La Libertad con 45 aerogeneradores, 'Talara' en Piura con 17 aerogeneradores, 'Marcona' en Ica con 11 aerogeneradores y 'Tres Hermanas' en Ica con 33 aerogeneradores. Ya se están desarrollando otros proyectos para la construcción de nuevos parques eólicos con una capacidad de $162 \mathrm{MW}$ derivados del concurso hecho por el gobierno a comienzos del 2016.

Otra fuente de aprovechamiento de energía es la energía solar, que ya la aprovechamos desde hace años en termas solares, autos solares y en pequeñas aplicaciones como calculadoras solares. En este caso la nueva aplicación en el Perú es la construcción de parques solares para la generación de electricidad en gran escala para las poblaciones y las industrias. Desde el año 2012 han entrado en operación comercial cinco parques solares en el sur del país, en donde se tiene la mayor radiación solar anual y también la más limpia. Estos parques son: 'Majes' en Arequipa (22 MW), 'Repartición Solar' en Arequipa (22 MW), Panamericana Solar en Moquegua (20 MW), 'Moquegua FV' en Moquegua (19 MW) y 'Tacna Solar' en Tacna (20 MW). En total estos parques solares aportan $103 \mathrm{MW}$ al sistema interconectado nacional del sur del país. Estos parques, dependiendo de la tecnología empleada, tienen de 56 mil a 76 mil paneles solares cada uno que se encargan de transformar la energía solar en electricidad y, por la cantidad de paneles usados, ocupan un área de 100 a 120 hectáreas. Al igual que en el caso de la energía eólica, el gobierno efectuó un concurso a inicios del 2016, por lo que ya se están desarrollando otros proyectos para la construcción de nuevos parques solares con una potencia total instalada de $184 \mathrm{MW}$. 


\section{ROCA FOSFÓRICA}

Esta es otra gran riqueza peruana que ha empezado a ser explotada comercialmente en la última década. En Bayovar, costa piurana de la provincia de Sechura, se descubrieron a mediados del siglo pasado unos inmensos yacimientos de roca fosfórica. Luego de un concurso público internacional efectuado en el año 2005 la transnacional Vale do Río, de Brasil, a través de su filial peruana, obtuvo la concesión del área 'Bayovar 2' por 27 años. A mediados del 2010 Vale empezó la exportación de concentrados de fosfatos que tienen $29 \%$ de P2O5 y desde ese año la estadística es la siguiente:

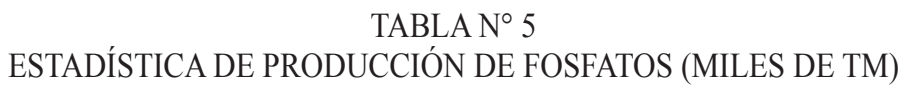

\begin{tabular}{|c|c|}
\hline AÑO & PRODUCCIÓN \\
\hline 2010 & 1134 \\
\hline 2011 & 8889 \\
\hline 2012 & 10338 \\
\hline 2013 & 14832 \\
\hline 2014 & 10868 \\
\hline 2015 & 11146 \\
\hline
\end{tabular}

Fuente: MINISTERIO DE ENERGÍA Y MINAS.

Estadisticas de producción de minerales no metálicos. Lima, 2016.

La explotación de este recurso natural es sencilla: debido a que la roca fosfórica está a solo unos metros debajo de la capa de suelo del desierto y de los bosques secos piuranos, no hay necesidad de dinamitar el suelo, solo excavar. Por estas características ya hay otras empresas internacionales interesadas en explotar otras áreas del gran yacimiento. Considero que aquí se debe dar también la ansiada industrialización del país con énfasis en fertilizantes. Así, una parte de este concentrado de fosfatos debería quedarse en Piura para procesarlos y obtener fertilizantes que no producimos y que tenemos que importar, como es el caso de los superfosfatos, fosfato monoamónico y fosfato diamónico. Se generaría entonces un polo de desarrollo especializado en fertilizantes, debido a que la costa piurana produce también gas natural que es la materia prima para producir urea, un fertilizante nitrogenado intensamente usado por los campos agrícolas a nivel mundial y que tampoco producimos en el Perú. La energía eléctrica que requieren estos proyectos se está obteniendo con los proyectos de parques eólicos del norte que ya están operando, como Cupisnique en La Libertad y Talara en Piura, en 
adición a la electricidad producida en las grandes hidroeléctricas de Poechos, Olmos y Gallito Ciego en Piura y Lambayeque.

\section{CONCLUSIONES}

1. El precio internacional de los metales, el descenso de la producción china y la situación europea, entre otros, ha influido en la producción minera del Perú y en la economía de nuestro país en la década 2006-2016. Asimismo en esta década no se aprovechó los años de bonanza iniciales y no se dio un mayor valor agregado a nuestra producción minera. En todos estos años hemos tenido conflictos con las comunidades con un factor común: la preocupación de las poblaciones locales respecto al comportamiento social de las empresas mineras, a la situación del recurso agua y la contaminación del ambiente.

2. En forma paralela a la primera década de funcionamiento del Programa de Estudios Básicos, la industria petrolera ha visto descender aún más la producción de petróleo, que está en manos privadas, desde 77,500 B/D en el 2006 hasta 48,820 B/D en agosto del 2016, continuando la caída de la producción que empezó en la década de 1990. Se espera que Petroperú retome esta actividad, que se concluya el proyecto de modernización de la refinería de Talara y que las nuevas unidades de refinería La Pampilla entren en operación exitosamente para disponer de combustibles de mejor calidad con menos azufre.

3. Durante los años 2006-2016 no se han impulsado proyectos industriales para aprovechar el gas y los líquidos de Camisea. La industrialización en base a la petroquímica del gas es clave para el desarrollo económico del país. La Ley 26221 de 1993, que establece que los propietarios de los hidrocarburos son los que tienen el contrato de licencia, debe cambiarse para que el Estado tenga soberanía sobre este recurso. Actualmente los operadores internacionales de Camisea privilegian la exportación del gas antes que su uso en la industrialización nacional (petroquímica, química y fertilizantes).

4. En energías renovables la última década ha visto el inicio de la producción de energía eléctrica con cuatro proyectos de energía eólica y cinco proyectos de energía solar que ya están en operación comercial y que, en conjunto, están aportando $342 \mathrm{MW}$ de electricidad al sistema interconectado nacional. Asimismo, este año se ha concursado la instalación de otros parques eólicos y solares por un total de $346 \mathrm{MW}$ de generación eléctrica. Se requiere que estos proyectos continúen para aprovechar el gran potencial de nuestro país en estas 
energías limpias que no requieren la quema de combustibles para producir electricidad ni poner en acción a la energía nuclear.

5. En la década 2006-2016 se ha iniciado de la explotación de los grandes yacimientos de roca fosfórica de Bayovar, Piura. No se han generado problemas de producción ni de índole social. Otras empresas internacionales desean ingresar a Bayovar para explotar nuevas áreas. Es necesario que se desarrolle una política nacional orientada al aprovechamiento de la roca fosfórica mediante su industrialización, para la producción de fertilizantes fosfatados que actualmente importamos. Debido a que más al norte, en Talara, se produce gas natural que es la materia prima para producir urea, se podría desarrollar en la zona un polo industrial de producción nacional de fertilizantes.

\section{REFERENCIAS BIBLIOGRÁFICAS}

Campodónico, H. (2016) . Enero 25. Tenemos que ser los dueños de la molécula. La República, Lima, p. 5.

Ministerio de Energía y Minas. (2008). Atlas Eólico del Perú. Lima: MINEM/ Banco Mundial.

(2013) Boletín Estadístico de Minería, Reporte Anual. Lima: MINEM.

(2016) Estadísticas de producción de minerales no metálicos. Lima: MINEM.

Perupetro (2016). Estadística Petrolera. Lima: Perupetro.

Tamayo, J.; Salvador, J.; et al. (Edit.). (2014). La industria del gas natural en el Perú. A diez años del Proyecto Camisea. Lima: Osinergmin. 